\title{
Estimate of JAK 2 V617F Mutation and Activity of Catalase Enzyme in Myeloproliferative Neoplasm
}

\author{
Heba Rushdi Abdullah, Perry Habib Saifullah, Ziad Ahmed Shebib and Ahmed Rushdi Abdullah \\ Department of Cytogenetic, Environmental Pollutants and Genetic Impacts Centre, Ministry of \\ Science and Technology, Baghdad-Iraq. \\ Department of Chemistry, College of Science for Women-Baghdad University, Baghdad-Iraq. \\ Department of Immunology,National Centter of Heamatology, Al-Mustansiriya University, Iraq. \\ Department of Microbiology, Medical College, Al-Iraqia University, Baghdad-Iraq.
}

\begin{abstract}
Background: Myeloproliferative neoplasms (MPNs) are a range of clonal hematological diseases with overlapping features, a specific mutation in the JAK2 gene, which encodes a tyrosine Kinase has been shown to be associated with the myeloproliferative phenotypes (PV, ET and PMF). Aim: Estimate the proportion of JAK2 V617F mutant gene and estimate the significance of antioxidant enzyme (Catalase) is mainly associated with increased oxidative stress this is resulting increase of the free oxygen radicals and relate it with suspected myeloproliferative neoplasms (MPNs) in Iraqi patients. Materials and Methods: Total of (51) patients with suspected myeloproliferative neoplasms (MPNs) and 20 healthy individuals were analyzed for the JAK2 V617F mutation. After DNA extraction, detection of the mutation was done using (ARMS) PCR amplification, activity of Catalase was measured spectrophotometrically. Results: Of 51 patients, the JAK2 V617F mutation (V617F) was detected in 33 out of 42, with PV (81\%), and four of patients with ET and PMF (40\% and $50 \%$, respectively). The prevalence of this mutation is more associated with male than female about $(62 \%)$. Catalase activity was found highly significant $(\mathrm{P}<0.01)$ among suspected MPN patients when compared with control group. Conclusion: JAK2 V617F mutation screening can be incorporated in the initial estimate of patients suspected of having MPNs. Catalase enzyme used a biomarker of enzymatic alteration in MPN Iraqi patients.
\end{abstract}

Keywords: JAK2 ${ }^{\mathrm{V} 617 \mathrm{~F}}$ mutation, myeloproliferative neoplasms, Catalase activity.

\section{Introduction}

Myeloproliferative neoplasms (MPNs) are a range of clonal hematological diseases with overlapping features. There are three main entities are polycythaemia Vera (PV), essential thrombocythemia (ET) and primary myelofibrosis (PMF), that are a produces too much of a particular type of blood cell [1]. In 2008 the World Health Organization (WHO) defined MPNs as cancers, but this is an issue of definition, doesn't have any specific effect on your treatment or prognosis [2]. People diagnosed by a myeloproliferative neoplasm (MPN) worldwide, with incidence rates 3.1 per 100,000 year [3], while in Iraqi national center of haematology 250 cases within 2014. myeloproliferative neoplasm (MPN) are a clonal excess hematopoiesis stem cell and this disease characterized by proliferation of one or more of the myeloid lineages in the bone marrow, then resulting the an elevated red blood cell count, or white blood cell or platelet count in the peripheral blood, and presence/ absence of Palpable enlargement of the spleen (splenomegaly) [4],[5]. And it supports this diagnostics by molecular factor; JAK2 $2^{\mathrm{V} 617 \mathrm{~F}}$ mutation is present in more than $95 \%$ of patients with PV and more than $50 \%$ of patients with PMF and ET [6].

On the other hand, Reactive Oxygen Species (ROS) are accumulate in MPN patients, resulting in an increase in oxidative DNA damage so caused the decreased one of antioxidant enzymes; catalase [7]. The present study, we evaluated the prevalence of the JAK2 V617F mutation and its clinical laboratory correlations with catalase (CAT) activity in patients with MPNs and in healthy control.

\section{Material and Methods \\ Patients:}

A patient are (51) screened of having MPN that were referred by physicians for JAK2 V617F mutation analysis. who were attending to (Iraqi national center of hematology/ 
Almustansiriyah University) from beginning January 2015 up to the June 2015, age were ranged (30-72) years, with 32 males and 19 females, Questioner diagnosis of MPN was depend on regards of age, sex, complete blood picture, smoking, patient's family history of leukemia, previous disease, blood section, physical examination include abdominal examination for palpable spleen and liver, and didn't receive any treatment. (Medical data were collected through direct interview with the patients).

\section{Blood samples:}

Five $\mathrm{ml}$ of blood was took from every patient and placed in (activator clotting tubes), after allowing the blood to clot at room temperature, Granulocyte was separated and placed in EDTA tube for DNA extraction by using ready kit (The Wizard® Genomic DNA Purification Kit/promega/USA), and performed JAK2 mutation test to detect this mutation on genomic DNA (ARMS-PCR). Patients and healthy control's sera were screened for the catalase activity.

\section{ARMS- PCR for the detection of JAK2 V617F mutation:}

Tetra Primer Amplification Refractory Mutation Screening Polymerase Chain Reaction ARMS-PCR an extremely efficient detection method of single nucleotide polymorphisms (SNPs). This method consists of two pairs of primers to amplify wild type and mutant respectively; forward outer primer (FO), reverse outer primer (RO), forward inner wild type primer (FWT), and reverse inner mutant specific primer (RWT) were used. Two amplification allele-specific reactions occur in opposite directions, simultaneously. In the presence of the JAK2 mutation the reverse inner mutant specific primer and the forward outer primer bind to produce a fragment of $279 \mathrm{bp}$. In the presence of wild-type JAK2 the reverse outer primer and the forward inner wild-type specific primer bind to produce a fragment of $229 \mathrm{bp}$. The forward primer from one site and the reverse primer from the other generate a control fragment of $463 \mathrm{bp}$ in all cases [8].
ARMS-PCR amplification of the JAK2 V617F mutation was done by common reverse PCR Tetra primers (IDT, Bulgarian) specific for the JAK2 V617F gene; primers sequences, melting temperature were listed in Table (1). The amplification of JAK2 V617F Mutation Gene was performed in a PCR -Thermo Xp Cycler and the reaction mixture was prepared according to the procedure that suggested by the manufacture company (Promega, UAS), within a total volume of $25 \mu \mathrm{L}$ containing $2 \mu \mathrm{l}$ (25-1250 ng) DNA, $12.5 \mu \mathrm{L}$ of Go Taq ${ }^{\circledR}$ Green Master Mix, 2x (Promega, UAS), $1 \mu$ l of each $\mathrm{FO}, \mathrm{RO}$ and Fwt, and Rmt primer with adding $6.5 \mu \mathrm{l}$ of nuclease free water to get final volume of $25 \mu \mathrm{l}$. the PCR program was applied according to (Promega, UAS), on the thermal cycler (C1000 thermal cycler, BIORAD, UAS). The cycling conditions as follows: an initial denaturation step at $94^{\circ} \mathrm{C}$ for $6 \mathrm{~min}$, followed by 40 cycles of denaturation at $94^{\circ} \mathrm{C}$ for $40 \mathrm{sec}$., annealing at $56^{\circ} \mathrm{C}$ for 45 sec., extension at $72^{\circ} \mathrm{C}$ for $45 \mathrm{sec}$., and a final extension step of $10 \mathrm{~min}$ at $72^{\circ} \mathrm{C}$ [9].

A total of $10 \mu \mathrm{l}$ from the amplified PCR products along with $100 \mathrm{bp}$ Ladder were run on $1 \%$ agarose gel electrophoresis at 10 Volts for $30 \mathrm{~min}$, then the fragments were visualized by ethidium bromide $(0.5 \mu \mathrm{g} / \mathrm{mL})$ under UV Trans illuminator Scope 21. 


\begin{tabular}{||c||c|c|}
\hline \multicolumn{1}{|c||}{ Primers } & Nucleotide sequences $\left(5^{\prime} \longrightarrow \mathbf{3}^{\prime}\right)$ & $\mathbf{T m}^{\prime} \mathbf{C}^{\circ}$ \\
\hline \hline Forward Outer(FO) & 5' TCCTCAGAACGTTGATGGCAG 3' $^{\prime}$ & 54 \\
\hline \hline Reverse Outer(RO) & 5' $^{\prime}$ ATTGCTTTC CTTTTTCACAAGAT 3' & 48 \\
\hline \hline Forward inner Wild Type (Fwt) & 5'GCATTTGGTTTTAAATTATGGAGTATATG 3' $^{\prime}$ & 52.9 \\
\hline \hline Reverse inner mutant specific primer (Rmt) & 5' $^{\prime}$ GTTTTACTTACTCTCGTCTCCACAAAA 3' & 55.1 \\
\hline
\end{tabular}

\section{Measurement of Catalase Activity Assay Principle}

Catalase activity was measured spectrophotometric ally by following at 240 nm. [10]

$$
2 \mathrm{H}_{2} \mathrm{O}_{2}-\text { catalase } \longrightarrow \mathrm{O}_{2}+2 \mathrm{H}_{2} \mathrm{O}
$$

\section{Reagents Preparation:}

These reagents prepared freshly before measurement:

A. Phosphate buffer (0.05 M; pH 7.0):

Reagent A: Na2HPO4 $0.05 \mathrm{M}$ solution and $0.05 \mathrm{M} \quad \mathrm{KH} 2 \mathrm{PO} 4$ solution were mixed to made a $\mathrm{pH}$ solution $=7.0$.

B. Hydrogen peroxide $\left(\mathrm{H}_{2} \mathrm{O}_{2}\right)$ solution (0.059 M; pH 7.0):

Reagent B: $0.6 \mathrm{ml}$ of $30 \% \mathrm{H}_{2} \mathrm{O}_{2}$ was added to $100 \mathrm{ml}$ of phosphate buffer. Sample: Immediately prior to use dilute the enzyme in $0.05 \mathrm{M}$ phosphate buffer (Reagent $\mathbf{A}$ ), $\mathrm{pH} 7.0$ to obtain a rate of 0.03-0.07 $\Delta \mathrm{A} / \mathrm{min}$.

\section{Procedure:}

This test was done by UVSpectrophotometer 1800 at $\left(25^{\circ} \mathrm{C}\right)$, Filly $0.059 \mathrm{M}$ of Reagent $\mathbf{B}$; Hydrogen peroxide were placed in sample tube, and followed with and $1.9 \mathrm{ml}$ of Reagent grade water. The reaction was started by adding 0.1 $\mathrm{ml}$ of diluted enzyme to sample tube tubes were mixed and incubated at $25 \mathrm{C}^{\circ}$ for (4-5) minutes in Spectrophotometer, and then record the decrease of absorbance for (2-3) minutes against blank (Reagent $\mathbf{A}$; Phosphate buffer instead of sample $\left(\Delta \mathrm{A}_{0}\right)$ at $240 \mathrm{~nm}[11]$.

\section{Calculation:}

Activity can be calculated by using the following formula: Volume activity

$$
(\mathrm{U} / \mathrm{ml})=\frac{\left(\Delta A_{S}-\Delta A_{0}\right)}{(0.0436)} \times \frac{(3.0 \mathrm{ml})}{(0.1 \mathrm{ml})} \times(\mathrm{DF})
$$

$\Delta \mathrm{A}_{\mathrm{S}}-\Delta \mathrm{A}_{0}=$ Difference between initial absorbance and after (3) min. final absorbance.

$0.0436=$ Mille molar extinction coefficient $(\varepsilon)$ of hydrogen peroxide at $240 \mathrm{~nm}(\mathrm{~cm} 2 / \mu \mathrm{mol})$.

DF $=$ Dilution Factor

$0.1=$ Volume (in milliliter) of diluted enzyme used.

\section{Statistical Analysis}

Data was analyzed using Statistical Package for the Social sciences (SPSS, version 18). Quantitative variables like Age, gender, splenomegaly, WBC, RBC, $\mathrm{Hgb}, \mathrm{PCV}$, and Platelets were presented with mean \pm SE.

Student's t-test was used to compare means of Catalase activity. A p-value $<0.05$ was considered statistically significant, while $\mathrm{P}$-value $<0.01$ was considered statistically highly significant.

\section{Results}

The JAK2 V617F mutation was analysed in some Iraqi patients with suspected MPNs that were referred by physicians for JAK2 V617F mutation analysis. In this study, A total of 51 subjects with MPNs; $37(72.5 \%)$ of the patients were positive for the JAK2 V617F mutation, whereas $14(27.4 \%)$ patients were negative for the JAK2 V617F mutation. detected by an amplification refractory mutation screening (ARMS) PCR. The JAK2 V617F mutation-positive group (37 patients) included $33(81.01 \%)$ patients with PV, $2(50 \%)$ patients with ET, 2 (50\%) patients with primary myelofibrosis (PMF). The JAK2 V617F mutation-negative group (14 patients) included $9(20.02 \%)$ patients with PV, 3 $(50 \%)$ patients with MF, 2 (60\%) 
patients with ET. Among 51 patients; the mean age of the patients was $52.5 \pm$ 2.24 (Mean + SE) years, with ranged from 30 to 72 years, there were 32 males $(62.8 \%)$ and 19 females $(32.2 \%)$ with a male to female ratio of $1.7: 1$.

Splenomegaly was present in 22 patients $(44 \%)$. These results are showed

Table (2)

Distribution of the studied groups was according to age, gender and Splenomegaly.

\begin{tabular}{|c|c|c|c|c|c|c|}
\hline \multicolumn{2}{|c|}{ Parameters } & \multicolumn{2}{|c|}{ MPNs Group No.=51 } & \multicolumn{2}{|c|}{ Control group No. $=20$} & P. value \\
\hline \multicolumn{2}{|c|}{$\begin{array}{c}\text { Age } \\
\text { Mean } \pm \text { SE }(\text { Range })\end{array}$} & \multicolumn{2}{|c|}{$\begin{array}{c}52.5 \pm 2.24 \\
(30-72) \\
\end{array}$} & \multicolumn{2}{|c|}{$\begin{array}{c}44.22 \pm 2.24 \\
(30-61)\end{array}$} & $0.015^{*}$ \\
\hline \multirow{3}{*}{ Gender } & Tol & No. & $\%$ & No. & $\%$ & \multirow{3}{*}{0.232} \\
\hline & Vlale & 32 & $62.8 \%$ & 8 & $40 \%$ & \\
\hline & Female & 19 & $37.2 \%$ & 12 & $42.8 \%$ & \\
\hline Splenomegaly & Yes & 22 & $44 \%$ & & & --- \\
\hline
\end{tabular}

Note: *: significant using student $T$ - test at $>0.05$ level of significance.

The results of blood cell indices for MPN group and control group as apparent in Table (3). The current mean was showed significant differences $(\mathrm{P}>0.05)$ in (WBC, PLAT count), whereas (RBC, PCV, HGB) revealed

Table (3)

Haematological parameters for MPN group and control group.

\begin{tabular}{|c|c|c|c|c|}
\hline \multicolumn{2}{|c|}{ Parameters } & MPN Group/ No.= 51 & Control group/ No. $=20$ & P. value \\
\hline \multirow{2}{*}{$\begin{array}{c}\text { WBC } \\
\left(\times 10^{9} / \mathrm{L}\right)\end{array}$} & Mean \pm SEM & $11.00 \pm 0.87$ & $6.44 \pm 0.29$ & \multirow{2}{*}{$0.012 *$} \\
\hline & Range & $(4.29-28.48)$ & $(4.2-9.79)$ & \\
\hline \multirow{2}{*}{$\begin{array}{c}\text { RBC } \\
\left(\times 10^{6} / \mu \mathrm{l}\right) \\
\end{array}$} & Mean \pm SEM & $6.10 \pm 0.25$ & $4.82 \pm 0.09$ & \multirow{2}{*}{$0.001 * *$} \\
\hline & Range & $(2.8-9.42)$ & $(4.26-5.27)$ & \\
\hline \multirow{2}{*}{$\begin{array}{l}\text { HGB } \\
(\mathrm{g} / \mathrm{dl})\end{array}$} & Mean \pm SEM & $15.57 \pm 0.52$ & $13.00 \pm 0.18$ & \multirow{2}{*}{$0.001 * *$} \\
\hline & Range & $(7.3-22.2)$ & $(12.4-14.7)$ & \\
\hline \multirow{2}{*}{$\begin{array}{l}\text { PCV } \\
(\%) \\
\end{array}$} & Mean \pm SEM & $48.27 \pm 1.43$ & $41.02 \pm 0.46$ & \multirow{2}{*}{$0.0001 * *$} \\
\hline & Range & $(15.4-69)$ & $(38.7-42.9)$ & \\
\hline \multirow{2}{*}{$\begin{array}{c}\text { PLAT } \\
\left(\times 10^{9} / 1\right)\end{array}$} & Mean \pm SEM & $405.80 \pm 48.00$ & $226.15 \pm 26.85$ & \multirow{2}{*}{$0.023 *$} \\
\hline & Range & $(83-1124)$ & $(180-323)$ & \\
\hline
\end{tabular}

Note: *: significant using student $T$ - test at 0.05 level of significance, **: $<0.01$ level of highly significance.

In this study, JAK2V617F mutation in peripheral blood of MPNs was demonstrated in Fig.(1). The internal control band in all cases is represented by a $463 \mathrm{bp}$ fragment, whereas the JAK2 V617F mutant is represented by a $279 \mathrm{bp}$ fragment, in the presence of wild type JAK2, specific primer is represented by a 229-bp fragment. highly significant differences $(\mathrm{P}>0.01)$ compared with control group. among MPNs group of patients when in Table (2). As for the mean age of with range (30-61), and male to female ratio was found $(0.66: 1)$. 


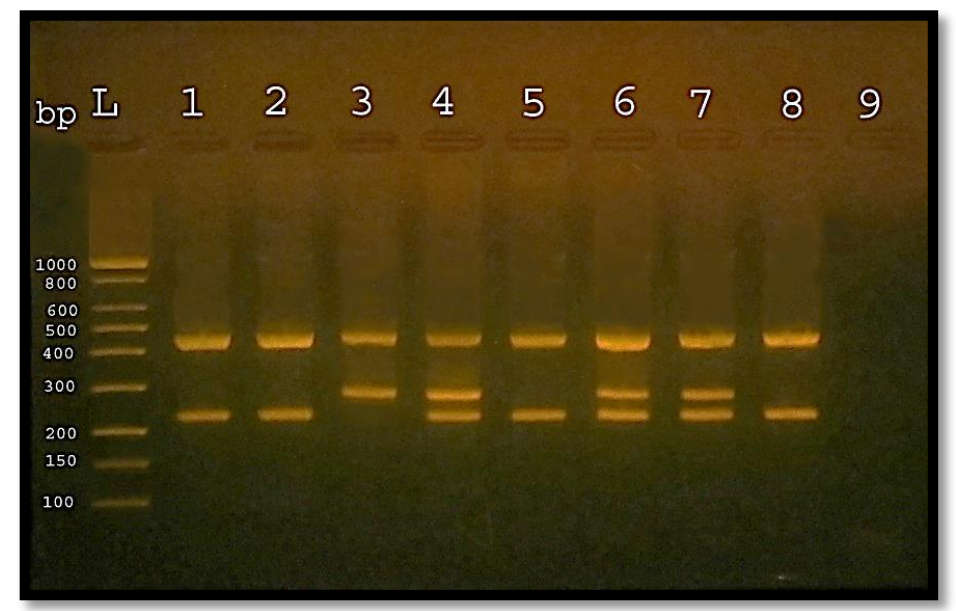

Fig.(1): Control band in 463 bp, amplified DNA in a 229 bp fragment, whereas the JAK2 V617F mutant in a 279 bp fragment, Lane L; 100 bp ladder. Lanes 1, 2, 5: show negative results (also pre, post treatment MPN), Lanes 3: show positive result (homozygous at 279bp), Lane 4, 6: show positive results (heterozygous at 279,229 bp), the lane 7: show positive control, Lane 8; show negative healthy control, while Lane 9; show distilled water. Use $0.5 \%$ agarose gel for 30 min. at 50 volt.

\section{Serum Catalase (CAT) Activity}

The results of present study showed that mean activity of catalase in a highly significant differences $(\mathrm{P}>0.01)$ among MPN group patients when compared with control group as shown that in Table (3.5) and Fig.(3.11).

\section{Table (4)}

Catalase enzyme activity among myeloproliferative neoplasm patients and healthy control groups.

\begin{tabular}{|c|c|c|c|}
\hline \multirow{2}{*}{ Parameter } & MPN Group/ No.= 51 & Control group/ No. $=20$ & P. value \\
\hline & Mean \pm SE & Mean \pm SE & \multirow{3}{*}{$0.0001 * *$} \\
\hline Catalase $(\mathrm{u} / \mathrm{ml})$ & $51.85 \pm 4.100$ & $112.02 \pm 3.01$ & \\
\hline Range & $(13.7-88)$ & $(90-134)$ & \\
\hline
\end{tabular}

Note: *: significant using student T- test at 0.05 level of significance, $* *:<0.01$ level of highly significance.

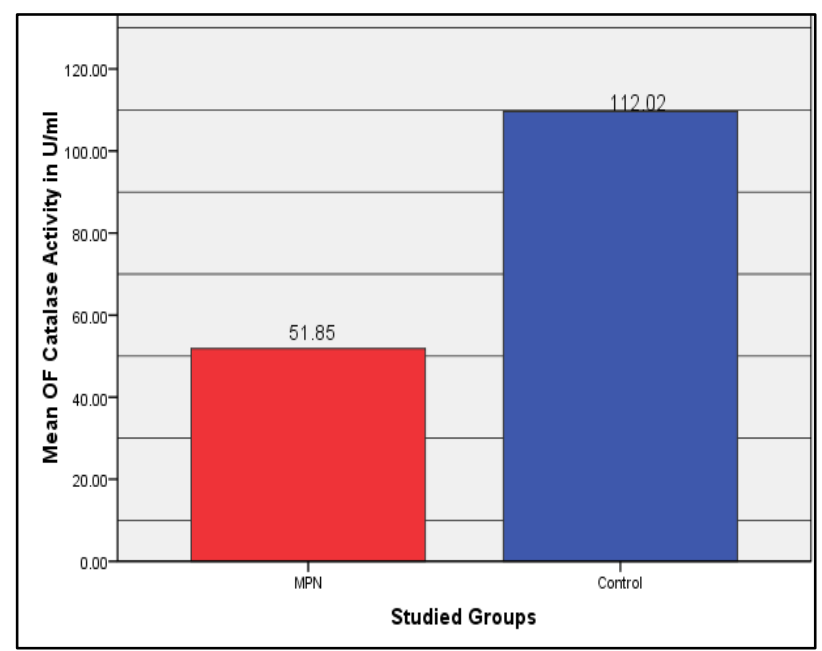

Fig.(2): Catalase activity in MPN group patients and control group. 


\section{Discussion}

Results from our study showed that a single somatic activating mutation JAK2-V617F was identified in the most of patients with $\mathrm{PV}$ and in about half of patients with ET or PMF. JAK2 V617F mutation is the first genetic indicator that is directly related with the pathogenesis of MPN [12]. For the same cause JAK2 V617F mutation has been included as an essential component (major criteria) in the 2008 WHO diagnostic criteria for PV, ET and PMF, and could be helpful in therapeutic targets, essentially when particular chemotherapeutic agents are used in the treatment of other cancers [13],[14].

Therefore, in this study, the JAK2 V617F mutation was identified in 51 patients with suspected MPNs that were referred by physicians for this mutation analysis. The vast majority of MPN patients with PV harboured the JAK2 V617F mutation (33 out of 42, corresponding to $81.01 \%$ ), this Finding is in agreement with Jones et al., Tefferi et al. \& Amy; who found JAK2 V617F mutation are present $(81 \%),(<90 \%)$ and $(82 \%)$ in $\mathrm{PV}$ patients Consecutive [8],[16],[17].

These differences could be explained with variable number of analyzed cases and the differences in sensitivity and specificity of methods (ARMS) applied for detection of JAK2- V617F mutation [17],[18].

This study showed the Amplification Refractory Mutation Screening (ARMS) PCR statement a single base was changed to be detected perfect PCR conditions. This is model for detection of the single base

$\mathrm{G} \rightarrow \mathrm{T}$ transversion connected with the JAK2 mutation, Also ARMS-PCR can indicate if a nonesuch was homozygous or heterozygous for the V617F JAK2 mutation it does not quantify the ratios of the wild type and mutant alleles, then ARMS enable differentiate between polymorphic alleles and very important in display more sensitivity to small amount of mutant DNA in a wild type background. This method have (20 to $30 \%$ ) of sensitivity (McLornan et al., 2006) [19], [20]. Splenomegaly was consider as one of diagnostic sign in suspected myeloproliferative neoplasms according to the WHO 2008 diagnostic criteria [12], The present result obviously demonstrates that (44\%) of MPN patients group had palpable spleen. This finding is going well with that by Mesa (2006), who mentioned that palpable spleen was seen in $(43.8 \%)$ of new diagnosis MPN [21].

Our study revealed that the MPN patients, displayed leukocytosis and higher levels of hemoglobin, hematocrit and platelets when compared with control group, as it was reported by previously published studies [22], [23].

Furthermore, we demonstrated that higher blood cell indices are associated with presence of JAK2-V617F mutation, a finding that indicates activated myelopoiesis [24]. In recent years, it has been demonstrated that superoxide anion as well as alkoxyl peroxyl and radicals could inactivate one of the antioxidant enzymes - catalase and reduce the effectiveness of cells to defend against free radical damage [25]. In addition Catalase is used by cells to defend against the toxic effects of hydrogen peroxide.

In this paper, it was shown that during MPN patients who have CAT activity is almost always low; these findings are interpreted according to the results of Paul (2011) who described these results by indicating a relationship between decreased catalase activities which may explain the ROS accumulation in MPN patients when compared with healthy people [26].

\section{Conclusion}

This study has presented the importance of peripheral blood mutation screening for JAK2 V617F by ARMS PCR can be incorporated in the initial evaluation of patients with suspected MPNs. The Catalase activity test was statistically highly significant between control group and the suspected MPN patients.

\section{Acknowledgement}

The authors would like to thank all the working staff of the National Center of Hematology for their help and support, special thanks for Dr. Ala'a F. Alwan, Miss. Baan Abdulatif Mtashar, Mrs. Marwah Hasan. 


\section{References}

[1] O. Visser a, A. Trama b, M. Maynadie' c, C. Stiller d, R. Marcos-Gragera e, R. De Angelis f, S. Mallone f, C. Tereanu b, C. Allemani b,i, U. Ricardi h, H.C. Schouten g, The RARECARE Working Group. Incidence, "survival and prevalence of myeloid malignancies in Europe" European Journal of Cancer, 48, 3257-3266, 2013.

[2] Christen Lykkegaard Andersen, Björn Andreasson, Hans Hasselbalch, Malin Hultcrantz, Håvar Knutsen, Marie Lindgren, Tove Skjelbakken, Jan Samuelsson, Thomas Stauffer Larsen. "Nordic guidelines on the diagnosis and treatment of patients with Myeloproliferative Neoplasms". The European Leukemia Net., 6 (1), 1-31, 2013.

[3] Tony Green, Jacob Grinfeld, Jyoti Evans, Will Thomas. "Myeloproliferative neoplasms (MPN)". Leukaemia \& Lymphoma Research; 20, 4-44, 2015.

[4] Johanason, P. "Epidemiology of the myeloproliferative disorders ptlycythemia Vera and essential thrombocythemia". Semin Thromb Hemost., 32,171-173, 2006.

[5] Barbui T, Barosi G, Grossi A, et al "Practice guidelines for the therapy of essential thrombocythemia. A statement from the Italian Society of Hematology, the Italian Society of Experimental Hematology and the Italian Group for Bone Marrow Transplantation". Haematologica 89,215-32, 2004.

[6] Brecqueville M, Rey J, Bertucci F, et al "Mutation analysis of ASXL1, CBL, DNMT3A, IDH1, IDH2, JAK2, MPL, NF1, SF3B1, SUZ12, and TET2 in myeloproliferative neoplasms" Genes, chromosomes \& cancer 51,743-55, 2012.

[7] Correa PN, Eskinazi D and Axelrad AA "Circulating erythroid progenitors in polycythemia vera are hypersensitive to insulin-like growth factor-1 in vitro: Studies in an improved serum-free medium" Blood 83, 99-112, 1994.

[8] Jones A.V, Kreil S, Zoi K, Waghorn K, Curtis C, Zhang L, et al. "Widespread occurrence of the JAK2 V617F mutation in chronic myeloproliferative disorders" Blood. 106(6), 2162-8, 2005.
[9] Nadali, F.; Ferdowsi, Sh.; Karimzadeh,P.; Chahardouli, B.; Einollahi, N.; Mousavi, S.A.; Bahar, B.; Dargahi, H.; Alimoghaddam, K.; Ghavamzadeh, A. and Ghaffari, S.H. "JAK2-V617F Mutation and Philadelphia Positive Chronic Myeloid Leukemia" IJHOSCR.3 (3), 43-45, 2009.

[10] Beers, R. Jr. and Sizer, I.W. "Measurement of Catalase activity" Journal of Biological Chemistry 195, 133-140, 1952.

[11] Bergmeyer, H. U. "Methods of Enzymatic Analysis" (3rd ed.), Blood, 3, pp. 273-286, 1983.

[12] Tefferi A, Vardiman JW. "Classification and diagnosis of myeloproliferative neoplasms: The 2008 World Health Organization criteria and point-of-care diagnostic algorithms". Leukemia; 22,1422, 2008.

[13] Bilici M, Tekin SB, Kandaz M, Cayır K, Ertekin MV, Ozmen HK, "The evaluation of the results of adjuvant chemo-radio therapy in patients with gastric cancer: results from a single center in eastern Anatolia" Turk J Med Sci; 42, 329-36, 2012.

[14] Cağlayan A, Akbulut Z, Atmaca AF, Altınova S, Kılıc M, Balbay MD, "Effects of neoadjuvant chemotherapy on pathological parameters and survival in patients undergoing radical cystectomy for muscle-invasive bladder cancer" Turk J Med Sci; 42, 623-9, 2012.

[15] Tefferi A. "Myeloproliferative neoplasms" The John M. Bennett 80th birthday anniversary lecture. Leukemia Research; 36, 1481-1489, 2012.

[16] Amy V. Jones, Sebastian Kreil, Katerina Zoi, Katherine Waghorn, Claire Curtis, Lingyan Zhang, Joannah Score, Rachel Seear, Andrew J. Chase, Francis H. Grand, Helen White, "Widespread occurrence of the JAK2 V617F mutation in chronic myeloproliferative disorders". Blood (First Edition Paper), blood, 1, 1-10, 2005.

[17] Xiao Z, Zhang Y, Li L, Nie L, Yang L, $\mathrm{Xu}$ S, "Haematologica" haematol., 6, 93787, 2008. 
[18] Tefferi A. Myeloproliferative neoplasms, "The John M. Bennett 80th birthday anniversary lecture". Leukemia Research; 36,1481-1489, 2012.

[19] Steensma D.P, Dewald GW, Lasho TL, Powell HL, McClure RF, Levine RL, et al. "The JAK2 V617F activating tyrosine kinase mutation is an infrequent event in both 'atypical' myeloproliferative disorders and myelodysplastic syndrome". Blood; 106(4), 1207-9, 2005.

[20] Donal McLornan, Melanie Percy, Mary Frances McMullin "JAK2 V617F: A Single Mutation in the Myeloproliferative Group of Disorders". Ulster Med J.; 75 (2) 112119, 2006.

[21] Mesa R.A, PowellH, Lasho T, Dewald GW, McClure R, Tefferi A. "A longitudinal study of the JAK2 (V617F) mutation in myelofibrosis with myeloid metaplasia: analysis at two time points". Haematologica. 91, 415-416, 2006.

[22] Ruben A. Mesa, Joyce Niblack, Martha Wadleigh, Srdan Verstovsek, John Camoriano, Sunni Barnes, Angelina D. Tan, Pamela J. Atherton, Jeff A. Sloan, Ayalew Tefferi., "The Burden of Fatigue and Quality of Life in Myeloproliferative Disorders (MPDs)". American Cancer Society; 109 (1), 2007.

[23] Srdan Verstovsek., "New Drugs Control Symptoms of Myeloproliferative Disorders and Improve Quality of Life for Patients" The University of Texas MD Anderson Cancer Center, OncoLog, 56, (1), 2011.

[24] C. Marty, C. Lacout, N. Droin et al., "A role for reactive oxygen species in JAK2 V617F myeloproliferative neoplasm progression," Leukemia. 27 (11), 21872195, 2013.

[25] Xu X, Zhang Q, Luo J, Xing S, Li Q, Krantz Sb, Fu X, Zhao Zj, "JAK2-V617F mutation "Blood, 109, 339, 2007.

[26] Paul Spencer Hole, Richard Lawrence Darley, and Alex Tonks, "Do reactive oxygen species play a role in myeloid leukemias". Blood., 117 (22), 2011.

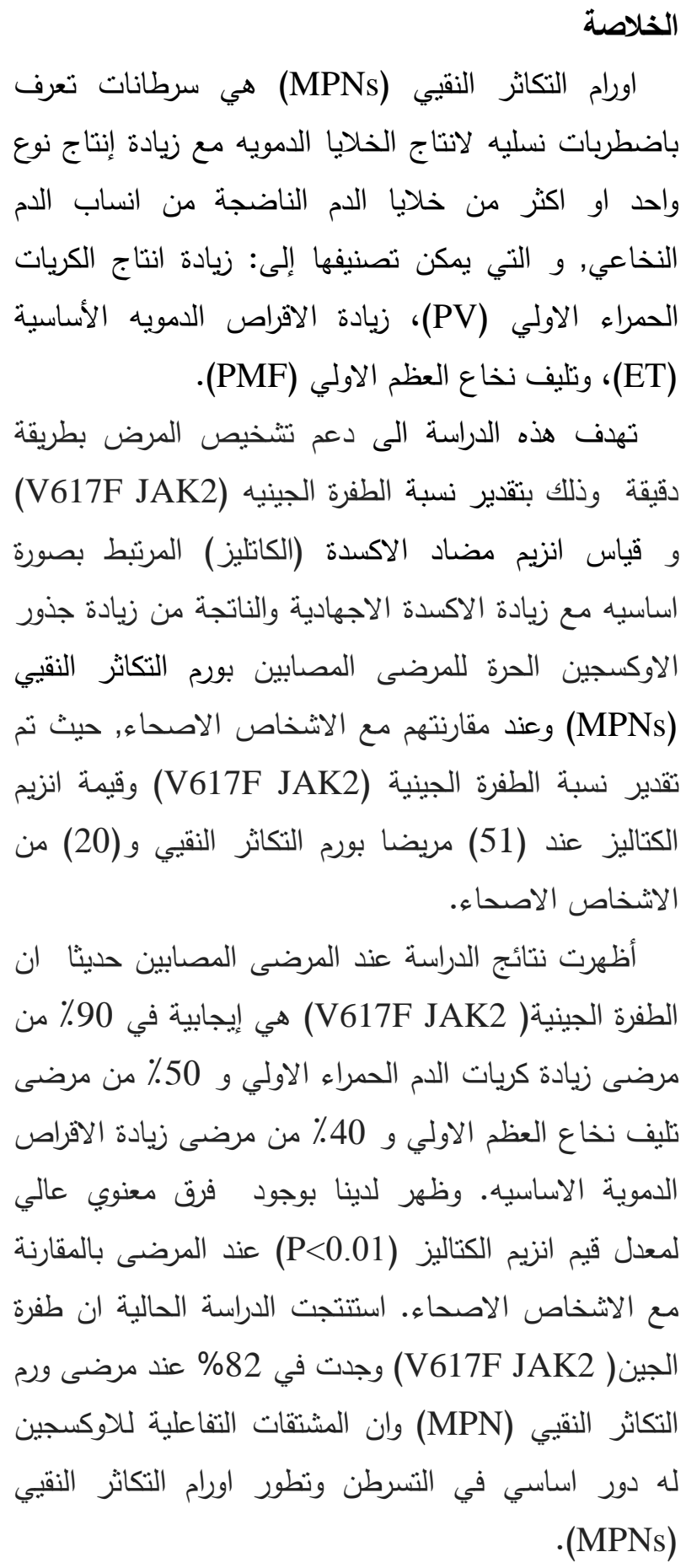

